\title{
The HST survey of the B2 sample of radio-galaxies: Optical nuclei and the FR I/BL Lac unified scheme^
}

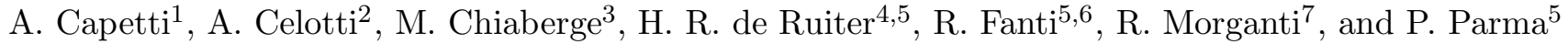 \\ 1 Osservatorio Astronomico di Torino, Strada Osservatorio 20, 10025 Pino Torinese, Italy \\ 2 SISSA/ISAS, Via Beirut 2-4, 34014 Trieste, Italy \\ 3 Space Telescope Science Institute, 3700 San Martin Drive, Baltimore, MD 21218, USA \\ 4 Osservatorio Astronomico di Bologna, Via Ranzani, 1, 40127 Bologna, Italy \\ 5 Istituto di Radioastronomia, Via Gobetti 101, 40129 Bologna, Italy \\ 6 Dipartimento di Fisica dell'Università di Bologna, Via Irnerio 46, 40126 Bologna, Italy \\ 7 Netherlands Foundation for Research in Astronomy, Postbus 2, 7990 AA, Dwingeloo, The Netherlands
}

Received 4 October 2001 / Accepted 3 December 2001

\begin{abstract}
We examine the optical properties of the nuclei of low luminosity radio-galaxies using snapshot HST images of the B2 sample. In agreement with the results obtained from the analysis of the brighter 3C/FR I sample, we find a correlation between fluxes (and luminosities) of the optical and radio cores. This provides further support for the interpretation that the optical nuclear emission in FR I is dominated by synchrotron emission and that accretion in these sources takes place in a low efficiency radiative regime. In the framework of the FR I/BL Lacs unified scheme, we find that the luminosity difference between FR I and BL Lac nuclei can be reproduced with a common beaming factor in both the radio and the optical band, independent of the extended radio luminosity, thus supporting such a scenario. The corresponding bulk Lorentz factor is significantly smaller than is expected from observational and theoretical considerations in BL Lacs: this can be interpreted as due to a velocity structure in the jet, with a fast spine surrounded by a slower layer.
\end{abstract}

Key words. galaxies: active - galaxies: elliptical and lenticular, cD - galaxies: jets - galaxies: nuclei

\section{Introduction}

The presence of a radio-source represents a common manifestation of nuclear activity associated to elliptical galaxies, in particular for the brightest members of this class. For example, among galaxies brighter than $M_{\mathrm{B}}<$ -21 , more than $20 \%$ have radio luminosities $L_{408 \mathrm{MHz}}>$ $10^{23.5} \mathrm{~W} \mathrm{~Hz}^{-1}$ (Colla et al. 1975). Due to the steepness of the radio luminosity function, most of them are low luminosity radio-sources and show the characteristic edge-darkened FR I radio morphology (Fanaroff \& Riley 1974). However, in observing bands other than the radio, emission related to their active nuclei remains largely elusive. Optical spectra of low luminosity radio-galaxies are in fact dominated by the stellar component of the host

\footnotetext{
Send offprint requests to: A. Capetti,

e-mail: capetti@to.astro.it

* Based on observations with the NASA/ESA Hubble Space Telescope, obtained at the Space Telescope Science Institute, which is operated by AURA, Inc., under NASA contract NAS 5-26555 and by STScI grant GO-3594.01-91A.
}

galaxy with only faint narrow emission lines, while permitted broad lines are seldom detected. Similarly, most of their X-ray emission originates in the ambient thermal plasma. With the limited information available, we cannot effectively constrain the physical properties and the emission processes at work in these AGNs, and to determine how these sources fit into the AGN unification schemes: one has to rely on the properties of the host galaxies, of the environment or of the extended radio structure.

Significant progress in our understanding of low luminosity radio-galaxies has been achieved recently thanks to high resolution HST and Chandra images, which enabled us to isolate their genuine optical and X-ray nuclear emission. In HST images unresolved nuclear sources are detected in the great majority of the $33 \mathrm{FR}$ I belonging to the 3CR sample (Chiaberge et al. 1999, hereafter CCC99). The optical flux density of these Central Compact Cores (CCC) shows a striking linear correlation with the radio core, arguing for a common non-thermal synchrotron origin. In five sources, for which it is possible to estimate the 
viewing angle based on the inclination of their circumnuclear dusty disks, the luminosity of the central source shows a suggestive dependence on the radio galaxy orientation, as qualitatively expected if the optical emission is indeed produced in a relativistic jet (Capetti \& Celotti 1999). The high rate of CCC detection suggests that a geometrically thick torus can be present at most in a minority of low luminosity radio galaxies. CCC fluxes also represent upper limits to any thermal/disk emission that translate (for a $10^{9} M_{\odot}$ black hole) into a fraction $\sim 10^{-7}-10^{-5}$ of the Eddington luminosity, suggesting that accretion takes place at low rate or in a low efficiency radiative regime.

This information also offers a new possibility of testing the FR I/BL Lac unification scheme, by directly comparing the optical nuclear properties of radio galaxies with their putative aligned (beamed) counterparts, analogously to the procedure followed in the radio band (Kollgaard et al. 1996). From this comparison Chiaberge et al. (2000b, hereafter CCCG00) found that the difference in luminosity (in radio and optical bands) between FR I nuclei and BL Lac is significantly smaller than would be expected, in the frame of a simple one-zone model, from the high bulk Lorentz factor of BL Lacs jets implied by observational and theoretical considerations (e.g. Dondi \& Ghisellini 1995; Ghisellini et al. 1998; Tavecchio et al. 1998). In order to reconcile these results with the unification scheme, they suggested that a velocity structure is present in the jet, with a fast spine surrounded by a slow layer, as already suggested by other evidence on larger scales (e.g. Laing 1993; Laing et al. 1999).

We recently obtained HST images for more than half of the B2 sample of low luminosity radio galaxies (see Capetti et al. 2000, hereafter Paper I). Therefore, it is now possible to complement the analysis performed for the $3 \mathrm{C}$ sources with a study of sources at lower radio luminosities. This extension will allow us to test the general validity of these results for the radio galaxy population and to explore in more detail the relationship between FR I and BL Lacs.

Thus in this paper we focus on the properties of their optical nuclei. In Sect. 2 we briefly present the properties of the B2 sample and the HST observations on which this study is based; the optical nuclear properties of the galaxies are described in Sect. 3 where we also quantify the contribution of their central optical sources. In Sect. 4 we discuss the implications of our results, which are summarized in Sect. 5. For consistency with Paper I we use $H_{0}=100 \mathrm{~km} \mathrm{~s}^{-1} \mathrm{Mpc}^{-1}$ and $q_{0}=0.5$.

\section{The sample and the HST observations}

The B2 sample consists of 100 low luminosity radiogalaxies. It is constituted by bright galaxies (down to a limiting magnitude $m_{\mathrm{v}}=16.5$ ) associated with radio sources from the B2 catalogue, which is complete to $0.25 \mathrm{Jy}$ at $408 \mathrm{MHz}$ (Colla et al. 1975; Fanti et al. 1978). The sample spans the power range between $10^{21}$ and $10^{26} \mathrm{~W} \mathrm{~Hz}{ }^{-1}$ at $1.4 \mathrm{GHz}$ with a pronounced peak around $10^{24} \mathrm{~W} \mathrm{~Hz}^{-1}$ and therefore gives an excellent representation of the radio source types encountered below and around the break in the radio luminosity function $\left(10^{24.5-25} \mathrm{~W} \mathrm{~Hz}^{-1}\right.$ at $408 \mathrm{MHz}$, Colla et al. 1975). Since it was selected at low radio frequency, the sample is largely unbiased for orientation. The B2 sources span a range in redshift between 0.002 and 0.176 .

HST images are available for 57 B2 radio galaxies. In the course of our HST snapshot program observations were obtained for 41 sources (see Paper I for details on the observations and data reduction). Each source was observed using two broad band filters, namely $F 555 \mathrm{~W}$ and $F 814 W$ (which approximately match the standard $V$ and $I$ filters) with an exposure time of 300 s. Public archival images for 16 additional objects were mostly obtained with the $F 702 W(R)$ filter (as part of the 3CR snapshot programs, De Koff et al. 1996; Martel et al. 1999).

While there is no bias in the selection of the sources that are part of the snapshot program (they were chosen randomly as far as their radio and optical properties are concerned), a bias might have been introduced by the inclusion of the 16 archive sources. However, the comparison of various parameters of the observed sub-sample with those of the sources that were not observed by HST shows that, except for a marginal (within $2 \sigma$ ) difference in redshift and radio power ${ }^{1}$, the sub-sample observed with HST can be considered as well representative of the whole B2 sample.

The data of the observed sources relevant for this paper are given in Table 1: in Col. 1 the B2 name, in Cols. 2 and 3 their alternative radio and optical identifications, in Col. 4 the redshift, in Cols. 5 and 6 the total and core radio power at $1.4 \mathrm{GHz}$, in Cols. 7 the optical nuclear flux density in the $I$ band and in Col. 8 the morphological classification of the extended radio emission.

\section{Detection and photometry of the Central Compact Cores}

With the aim of determining whether, superposed to the galaxy background, a CCC is present, we examined the nuclear brightness profile of the B2 galaxies. For the identification of optical cores we adopted the same procedure described in CCC99, i.e. a source brightness profile that, within 5 pixels from the center, shows a $F W H M$ consistent with the HST Point Spread Function $\left(\leq 0{ }^{\prime \prime} 08\right)$. For the bright elliptical galaxies harbouring the B2 sources this procedure is particularly effective as they all show well resolved central cusps characterized by shallow brightness gradients $\left(I(r) \sim r^{-\gamma}\right.$ with $\left.\gamma \sim 0-0.3\right)$. Figure 1 presents profiles for two sources in which we detected a nuclear source.

\footnotetext{
1 The median redshift and radio power of the two subsamples are $z=0.055_{-0.011}^{+0.008}, \log P_{\mathrm{t}}=24.05_{-0.03}^{+0.07}$ and $z=$ $0.067_{-0.007}^{+0.005}, \log P_{\mathrm{t}}=24.22_{-0.03}^{+0.06}$, respectively.
} 
Table 1. Nuclear properties of the sample.

\begin{tabular}{|c|c|c|c|c|c|c|c|}
\hline \multirow[t]{2}{*}{ Name } & \multicolumn{2}{|c|}{ Alternative ID } & \multirow[t]{2}{*}{ Redshift } & \multirow{2}{*}{$\begin{array}{l}\log P_{\mathrm{t}} \\
\mathrm{W} / \mathrm{Hz}\end{array}$} & \multirow{2}{*}{$\begin{array}{l}\log P_{\mathrm{c}} \\
\mathrm{W} / \mathrm{Hz}\end{array}$} & \multirow{2}{*}{$\begin{array}{c}\text { Optical } \\
\mathrm{erg} / \mathrm{s} \mathrm{cm}^{-2} \AA^{-1}\end{array}$} & \multirow[t]{2}{*}{ Radio Morph. } \\
\hline & Radio & Optical & & & & & \\
\hline $0034+25$ & & UGC 00367 & 0.0321 & 23.20 & 21.62 & Dusty & FR I \\
\hline $0055+26$ & 4C 26.03 & NGC 0326 & 0.0472 & 24.61 & 22.30 & $<1.0 \mathrm{e}-18$ & FR I \\
\hline $0055+30$ & & NGC 0315 & 0.0167 & 24.08 & 23.24 & $3.1 \mathrm{e}-17^{d}$ & FR I \\
\hline $0104+32$ & $3 \mathrm{C} 031$ & NGC 0383 & 0.0169 & 24.21 & 22.45 & $1.5 \mathrm{e}-17^{a, d}$ & FR I \\
\hline $0116+31$ & 4C 31.04 & & 0.0592 & 24.95 & 22.34 & Dusty & core \\
\hline $0120+33$ & & NGC 0507 & 0.0164 & 22.30 & 20.60 & $<0.6 \mathrm{e}-18$ & FR I \\
\hline $0149+35$ & & NGC 0708 & 0.0160 & 22.33 & 21.13 & Dusty & FR I \\
\hline $0648+27$ & & & 0.0409 & 23.62 & 23.00 & Dusty & core \\
\hline $0708+32$ & & & 0.0672 & 23.51 & 22.85 & $<1.5 \mathrm{e}-18$ & - \\
\hline $0722+30$ & & & 0.0191 & 22.67 & 22.02 & Spiral & disk \\
\hline $0755+37$ & 3C 189 & NGC 2484 & 0.0413 & 24.49 & 23.59 & $3.2 \mathrm{e}-17$ & FR I \\
\hline $0908+37$ & & & 0.1040 & 24.84 & 23.46 & $3.7 \mathrm{e}-18^{d}$ & FR I/II \\
\hline $0915+32$ & & & 0.0620 & 24.00 & 22.50 & Dusty & FR I \\
\hline $0924+30$ & & & 0.0266 & 23.52 & $<20.50$ & $<0.5 \mathrm{e}-18$ & FR I \\
\hline $1003+26$ & & & 0.1165 & 24.01 & 22.10 & $<0.2 \mathrm{e}-18$ & - \\
\hline $1003+35$ & $3 \mathrm{C} 236$ & & 0.0989 & 25.78 & 24.00 & $<3.5 \mathrm{e}-18$ & FR II \\
\hline $1005+28$ & & & 0.1476 & 24.25 & 22.60 & $<0.6 \mathrm{e}-18$ & FR I/II \\
\hline $1101+38$ & & MRK 421 & 0.0300 & 23.97 & 23.32 & $1.15 \mathrm{e}-14$ & core \\
\hline $1113+24$ & & & 0.1021 & 23.65 & $<22.50$ & $<0.2 \mathrm{e}-18$ & FR I/II \\
\hline $1204+34$ & & & 0.0788 & 24.47 & 22.88 & $<3.7 \mathrm{e}-18$ & FR II \\
\hline $1217+29$ & & NGC 4278 & 0.0021 & 21.24 & 20.48 & $2.4 \mathrm{e}-17$ & core \\
\hline $1251+27$ & 3C 277.3 & Coma A & 0.0857 & 25.37 & 22.95 & $1.5 \mathrm{e}-18^{a}$ & FR II \\
\hline $1256+28$ & & NGC 4869 & 0.0224 & 23.05 & 21.08 & Dusty & FR I \\
\hline $1257+28$ & & NGC 4874 & 0.0239 & 23.08 & 20.82 & $<0.2 \mathrm{e}-18$ & FR I \\
\hline $1321+31$ & & NGC 5127 & 0.0161 & 23.85 & 21.77 & Dusty & FR I \\
\hline $1322+36$ & 4C 36.24 & NGC5141 & 0.0175 & 24.55 & 22.21 & Dusty & FR I \\
\hline $1339+26$ & $4 \mathrm{C} 26.41$ & UGC 08669 & 0.0757 & 24.30 & $<22.40$ & Dusty & FR I \\
\hline $1346+26$ & 4C 26.42 & & 0.0633 & 24.55 & 23.37 & $3.4 \mathrm{e}-18$ & FR I \\
\hline $1347+28$ & & & 0.0724 & 24.05 & 22.34 & Dusty & FR I/II \\
\hline $1350+31$ & $3 \mathrm{C} 293$ & UGC 08782 & 0.0452 & 25.03 & 23.34 & Dusty & FR I/II \\
\hline $1357+28$ & & & 0.0629 & 24.03 & 22.45 & Dusty & FR I \\
\hline $1422+26$ & & & 0.0370 & 24.00 & 22.46 & $<6.9 \mathrm{e}-18$ & FR I \\
\hline $1430+25$ & 4C 25.46 & & 0.0813 & 24.20 & $<21.90$ & $<2.2 \mathrm{e}-18$ & FR I \\
\hline $1447+27$ & & & 0.0306 & 22.78 & 22.76 & $<4.1 \mathrm{e}-18$ & core \\
\hline $1450+28$ & & & 0.1265 & 24.50 & 23.01 & $<1.3 \mathrm{e}-18$ & FR I \\
\hline $1457+29$ & & & 0.1411 & 25.22 & $<23.40$ & Dusty & FR I \\
\hline $1455+28$ & 4C 28.38 & & 0.1470 & 24.89 & $<23.00$ & Dusty & FR II \\
\hline $1502+26$ & $3 \mathrm{C} 310$ & & 0.0540 & 25.36 & 23.40 & $3.5 \mathrm{e}-18^{a}$ & FR I \\
\hline $1511+26$ & $3 \mathrm{C} 315$ & & 0.1078 & 25.34 & $<24.24$ & Complex & FR I \\
\hline $1512+30$ & & & 0.0931 & 23.82 & 21.60 & $<0.4 \mathrm{e}-18$ & - \\
\hline $1521+28$ & $4 \mathrm{C} 28.39$ & & 0.0825 & 24.58 & 23.57 & $8.4 \mathrm{e}-18$ & FR I \\
\hline $1525+29$ & & UGC 09861 & 0.0653 & 23.98 & 22.04 & Dusty & FR I \\
\hline $1527+30$ & & & 0.1143 & 24.05 & 22.70 & $<2.1 \mathrm{e}-18$ & FR I \\
\hline $1553+24$ & & & 0.0426 & 23.57 & 23.02 & $1.4 \mathrm{e}-17$ & FR I \\
\hline $1557+26$ & & & 0.0442 & 22.81 & $<22.80$ & $<9.0 \mathrm{e}-18$ & core \\
\hline $1610+29$ & & NGC 6086 & 0.0313 & 22.93 & $<21.02$ & $<0.9 \mathrm{e}-18$ & FR I \\
\hline $1613+27$ & & & 0.0647 & 24.03 & 22.64 & $<1.5 \mathrm{e}-18$ & FR I \\
\hline $1615+32$ & $3 \mathrm{C} 332$ & & 0.1520 & 25.79 & 23.43 & $8.8 \mathrm{e}-17^{a}$ & FR II \\
\hline $1626+39$ & 3C 338 & NGC 6166 & 0.0303 & 24.49 & 23.00 & $1.0 \mathrm{e}-17^{a}$ & FR I \\
\hline $1658+30 \mathrm{~A}$ & $4 \mathrm{C} 30.31$ & & 0.0351 & 23.88 & 22.89 & $<1.1 \mathrm{e}-17$ & FR I/II \\
\hline $1726+31$ & $3 \mathrm{C} 357$ & & 0.1670 & 25.89 & 23.34 & $<0.9 \mathrm{e}-18$ & FR II \\
\hline $1827+32$ & & & 0.0659 & 24.07 & 22.95 & $<2.0 \mathrm{e}-18$ & FR I \\
\hline $1833+32$ & 3 C 382 & & 0.0578 & 25.07 & 23.85 & $4.8 \mathrm{e}-15^{a}$ & FR II \\
\hline $2116+26$ & & NGC 7052 & 0.0164 & 22.79 & 22.08 & $2.2 \mathrm{e}-18^{d}$ & FR I \\
\hline $2229+39$ & 3C 449 & UGC 12064 & 0.0181 & 24.03 & 22.11 & $1.8 \mathrm{e}-17^{a, d}$ & FR I \\
\hline $2236+35$ & & UGC 12127 & 0.0277 & 23.47 & 21.74 & $1.7 \mathrm{e}-18$ & FR I \\
\hline $2335+26$ & $3 \mathrm{C} 465$ & NGC 7720 & 0.0301 & 24.88 & 23.38 & $1.9 \mathrm{e}-17^{a}$ & FR I \\
\hline
\end{tabular}

${ }^{a}$ Flux in the $F 702 W(R)$ filter; ${ }^{d}$ central source in dusty galaxy.

Clearly, this method can only be applied when the central regions of the galaxy are not heavily affected by morphological peculiarities. Nuclear dust features are seen in 20 B2 sources (de Ruiter et al. 2001) and they prevent a detailed analysis of the innermost regions. Nonetheless in 5 of these galaxies the presence of central unresolved components is readily apparent from the visual inspection of the images and it is confirmed by the brightness profile behaviour; all of them are associated with disky dust structures. Two additional sources are excluded from the sample: B2 $0722+30$ which is a spiral galaxy and 


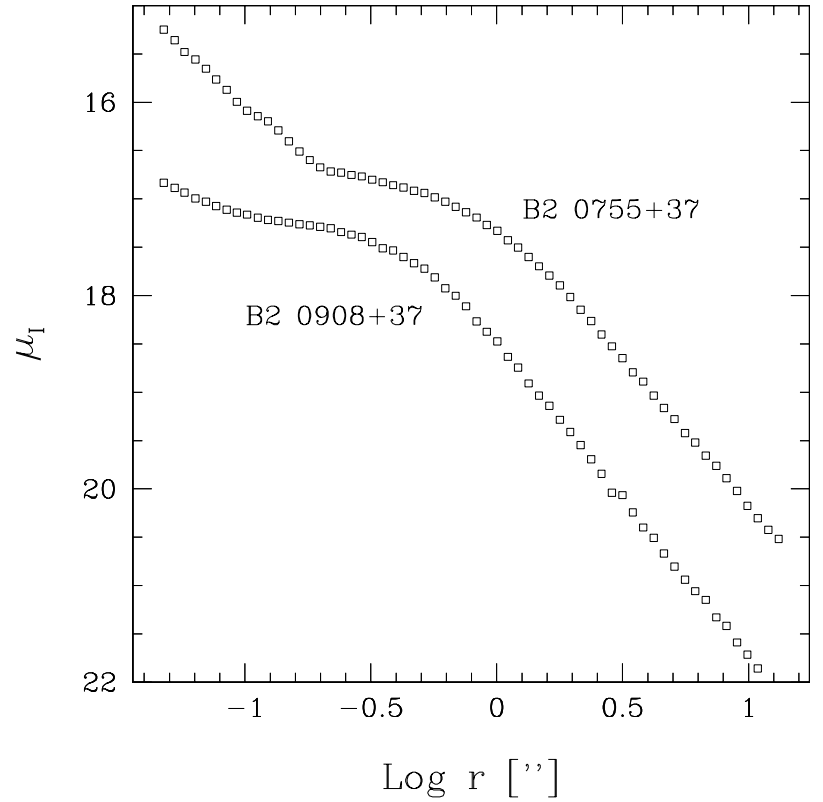

Fig. 1. Radial brightness profile for B2 $0755+37$ and B2 $0908+37$. Note the flat resolved galaxy core and the steepening of the profile caused by the nuclear source.

B2 $1511+26$ which presents a peculiar elongated morphology.

From the brightness profile analysis of the 35 remaining galaxies we found a CCC in 13 of them (for a total of 18 central sources). In the other 22 cases we set an upper limit to the CCC component defined, again following CCC99, as the light excess of the central $3 \times 3$ pixels with respect to the surrounding galaxy background. We also tested an alternative upper limit estimator, based on the subtraction of a Point Spread Function opportunely scaled until it produces an inversion in the galaxy profile, which yielded similar results.

Of the 18 unresolved optical sources, 2 are associated to B2 1615+32 (3C 332) and B2 1833+32 (3C 382), which are both Broad Line Radio Galaxies with a clear FR II radio morphology (Chiaberge et al. 2000a, 2001); as expected, we also detected a central source in B2 1101+38 (Mrk 421), a well known nearby BL Lac object. The remaining CCC are mostly associated to FR I sources (12), but we also found a CCC in 1 FR II, 1 FR I/II transition source and 1 core source. Six of them are in common with the $3 \mathrm{C} / \mathrm{FR}$ I sample (see Table 1 ).

We performed aperture photometry of the CCCs detected, adopting the internal HST/WFPC2 flux calibration. The resulting measurements are reported in Table 1 , with errors ranging from $5 \%$ to $25 \%$. Images in two broad filters are available for most galaxies, but the typical errors in the CCC photometry and the relatively small wavelength range available do not allow an accurate estimate of the optical spectral indices in most cases ${ }^{2}$.

${ }^{2}$ The spectral index error scales as $\sigma_{\alpha} \sim 8.5 \sigma_{F} / F$, where $\sigma_{F} / F$ is the fractional error on the flux estimate, and it amounts to typically $\sim 0.4-2$.

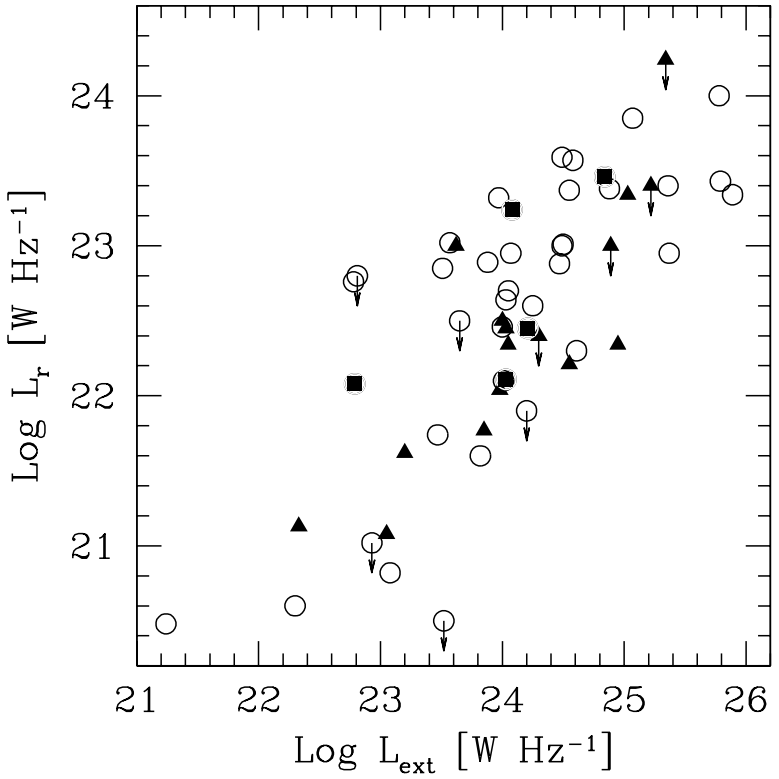

Fig. 2. Core versus extended radio luminosities at $1.4 \mathrm{GHz}$ of the B2 galaxies. Sources with obscured/complex optical morphology are marked as filled triangle, while dusty galaxies with a central source are filled squares; the remaining galaxies are plotted as circles.

\section{Results and discussion}

Before discussing the properties of B2 cores, it is important to establish the role of the sources with complex/obscured optical morphology for which we could not estimate the nuclear optical contribution, as their exclusion can in principle induce a significant bias in the final sample. This is particularly important for the B2/HST sample as the incidence of nuclear dust appears to be relatively high. In Fig. 2 we plot the radio core versus the extended radio luminosities for all B2 studied, marking the location of the complex/obscured galaxies with and without a central optical source. They are distributed over the whole range of extended luminosities and they do not show that radio cores are significantly different from the other galaxies with similar extended radio power. We quantitatively tested this result using the survival analysis package $A S U R V$ (Isobe \& Feigelson 1985, 1986). We derived the correlations between $L_{\mathrm{r}}$ (core) and $L_{\text {ext }}$ for the three classes separately and no significant differences are found. Thus, the exclusion of these sources will not affect significantly our results and conclusions, at least as far as the radio properties are concerned.

\subsection{The radio/optical plane for $B 2$ radio galaxies cores}

As discussed in the Introduction, the location of radio galaxies cores in the radio/optical plane is a very useful tool to study the properties of their nuclear emission. In Fig. 3 we plot the radio versus optical fluxes and luminosities of the CCC for the $40 \mathrm{~B} 2$ galaxies with CCC detection or upper limits together with the data derived for the 3C/FR I from CCC99. 

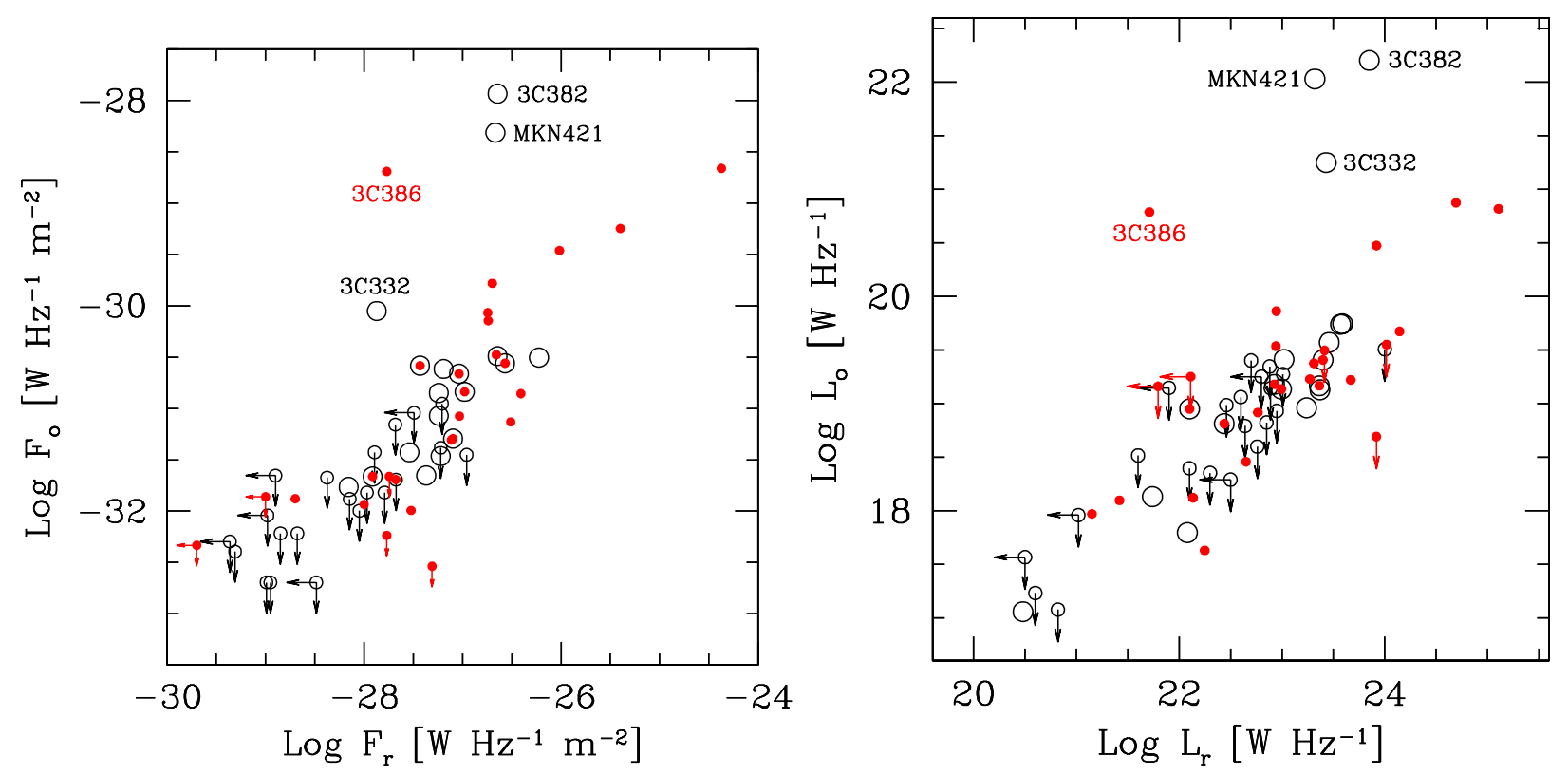

Fig. 3. Left panel: optical flux density of the CCC (at $8140 \AA$ ), versus radio core flux density (at $1.4 \mathrm{GHz}$ ). B2 sources are represented by empty circles (large for detections, smaller for upper limits), while the filled circles are the 3C/FR I sources from Chiaberge et al. (1999). Right panel: same as the left panel for the optical and radio luminosities. The three sources with the highest optical flux and luminosity are two BLRGs B2 1615+32 (3C 332) and B2 1833+32 (3C 382) and a BL Lac object B2 1101+38 (Mkn 421).

CCC in B2 sources are located essentially in the same region of the $F_{\mathrm{r}}-F_{\mathrm{o}}$ and $L_{\mathrm{r}}-L_{\mathrm{o}}$ planes as the $3 \mathrm{C}$ sources, and only avoid the upper right corner corresponding to the highest fluxes (and luminosities). The only B2 sources which significantly deviate are B2 1615+32, B2 1833+32 and B2 1101+38 which, as mentioned above, are two BLRG FR II and a BL Lac. This agrees with the results of Chiaberge et al. (2000a, CCCG00) that both BLRG and BL Lacs (in particular those belonging to the high energy peak class ${ }^{3}$, as it is for B2 1101+38) present a large optical excess with respect to FR I galaxies.

Radio and optical emission of the B2 cores are clearly correlated and a survival analysis gives a probability that they are extracted from a random population of only 0.0001. The best linear fit from ASURV has a slope of $0.8 \pm 0.1$ consistent with the result derived by CCC99 for the $3 \mathrm{C} / \mathrm{FR}$ I sample. It confirms the findings of the analysis by CCC99 and represents further evidence for a common synchrotron origin of the radio and the optical emission in FR I nuclei.

Galaxies for which we could only set upper limits to their optical nuclear emission also appear to be consistent with this correlation, but given their relatively high incidence, it is important to explore in more detail their nature. In the following we test the idea that their nuclei

\footnotetext{
${ }^{3}$ Instead of classifying BL Lacs according to their selection spectral band, we adopt the definitions of high and low energy peaked BL Lacs (HBL and LBL, respectively), which are based on the position of the (synchrotron) emission peak in the spectrum and therefore more indicative of the physical characteristics of the objects (Giommi \& Padovani 1994; Fossati et al. 1998).
}

follow a similar radio/optical correlation as the detected CCC, but their optical flux density is below our detection threshold. In this case, our ability to detect a CCC will depend primarily on its corresponding radio flux density, but also on its contrast with the host galaxy. Indeed sources with and without detected CCC are well separated in a plane in which we compare the radio core flux density with the galaxy central surface brightness (see Fig. 4). The separation occurs at an optical nuclear flux density (derived assuming a radio-to-optical spectral index of 0.7, as implied by the $L_{\mathrm{o}}-L_{\mathrm{r}}$ correlation) that exceeds the galaxy emission within the central $0.1^{\prime \prime} \times 0.1^{\prime \prime}$. Undetected CCC are thus likely to be simply too faint to be seen at the center of their host galaxies. This argues against alternative interpretations, such as, that they are obscured.

The inclusion of the $\mathrm{B} 2$ cores improves the coverage of the radio/optical plane with respect to the $3 \mathrm{C} / \mathrm{FR}$ I sample alone, in particular toward the lower luminosities. This region is particular important as here one might expect to see emerging the contribution of radiation processes other than synchrotron emission from the jet. The presence of any additional component, unrelated to the radio emission, should manifest itself as a flattening of the slope in the radio/optical plane. Clearly, this is not observed down to an optical luminosity of $\sim 10^{18} \mathrm{~W} \mathrm{~Hz}^{-1}$. For a $10^{9} M_{\odot}$ central black hole, this corresponds to a fraction $\sim 3 \times 10^{-8}$ of the Eddington luminosity in the optical band. As already remarked in CCC99, this is a clear evidence that accretion must occur at a very low rate and/or radiative efficiency.

Of the FR II and the transition FR I/II sources (which are represented by 7 and 5 galaxies, respectively) in 

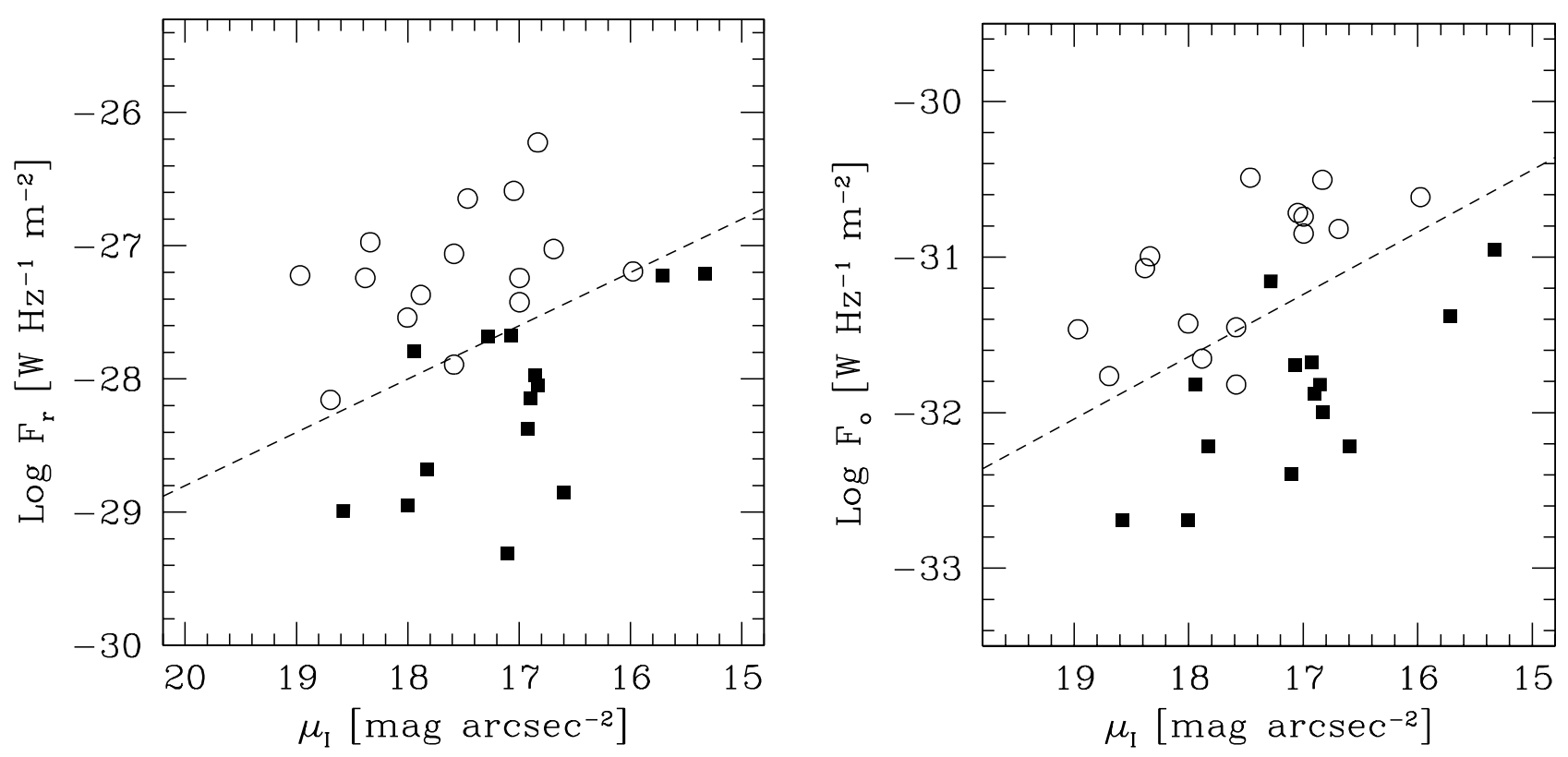

Fig. 4. Radio and optical core flux density versus central surface brightness of the host galaxy. Objects with detected optical sources (excluding the 2 BLRG and the BL Lac object) are marked with open circles, while squares represent galaxies with optical upper limits. The dotted line marks a constant ratio between optical core and galaxy emission (see text).

addition to the two BLRG already reported, only in two of them a CCC is detected and this clearly prevents any comparison between the properties of galaxies with different radio morphology.

\subsection{The FR I/BL Lacs unification model}

Measurements of the optical nuclear emission in low luminosity radio galaxies provided us with a new quantitative method of testing the FR I/BL Lac unification scheme. The comparison between the beamed sources and their putative counterparts must be restricted to objects of properties which do not depend on orientation. In particular, it is important that they share a common range of extended radio luminosity as this is plausibly linked to the energy carried by the relativistic jet, which is at the origin of the nuclear emission.

In Fig. 5 we plot the CCC optical and radio luminosity versus the extended radio luminosities. In this plane, the B2 sources significantly improve the coverage at low extended luminosities with respect to the $3 \mathrm{C} / \mathrm{FR}$ I sample studied by Chiaberge et al. (2000b). Indeed, in the $3 \mathrm{C} / \mathrm{FR}$ I there is only one object (3C 272.1) with $L_{\text {ext }}<10^{24} \mathrm{~W} \mathrm{~Hz}^{-1}$. Conversely, in the B2/HST sample, there are 21 galaxies below this value. In particular the B2 sources cover the region of extended radio power typical of HBL allowing a direct comparison over the whole range of the FR I/BL Lac populations.

To perform this comparison we consider BL Lacs selected from the 1 Jy catalogue (Stickel et al. 1991; Kühr et al. 1981) and the Einstein Slew survey (Elvis et al. 1992; Perlman et al. 1996), which comprise 34 and 48 objects, respectively. Of the 34 objects belonging to the $1 \mathrm{Jy}$ sample, 32 are classified as LBL and 2 as HBL, while of the 48 X-ray selected BL Lacs, 40 are HBL and 8 are LBL. The extended radio power is taken from Kollgaard et al. (1996) and Perlman et al. (1996). On average HBL are characterized by lower extended radio luminosities than LBL: the former sample extends from $\sim 10^{22}$ to $10^{24.5} \mathrm{~W} \mathrm{~Hz}^{-1}$, the latter from $\sim 10^{23} \mathrm{~W} \mathrm{~Hz}^{-1}$ to $10^{26} \mathrm{~W} \mathrm{~Hz}^{-1}$.

In the $L_{\text {ext }} / L_{\mathrm{o}}$ plane FR I nuclei and BL Lac lie in two well separated stripes and the regions covered by the two classes of BL Lacs are continuously connected. The optical luminosity of both BL Lacs and FR I increases with the extended power, with a similar logarithmic slope. Let us consider the $L_{\text {ext }} / L_{\mathrm{r}}$ plane. Radio galaxies follow a common trend, reflecting the well known correlation between core and extended radio emission (Giovannini et al. 1988). As expected, the two classes of BL Lacs appear to have different core radio powers (likely due to selection effects, e.g. Giommi \& Padovani 1994), but the logarithmic slope of the BL Lac population as a whole is similar to that measured in radiogalaxies. This is indeed what is expected if the difference in core luminosities between BL Lacs and FR I is due to relativistic beaming with a bulk Lorentz factor independent of $L_{\text {ext }}$.

It is clearly difficult to perform a detailed statistical comparison between these two classes of objects as they are affected by different selection biases. In particular, as discussed by Kollgaard et al. (1996), to adopt a survival analysis to account for the presence of upper limits, we would have to use different quantities (extended luminosities for radio galaxies and core luminosities for BL Lacs) as independent variables and the results cannot be directly compared. We preferred to follow their approach of evaluating the relationship between core and extended luminosities of the two classes by determining the bisector 

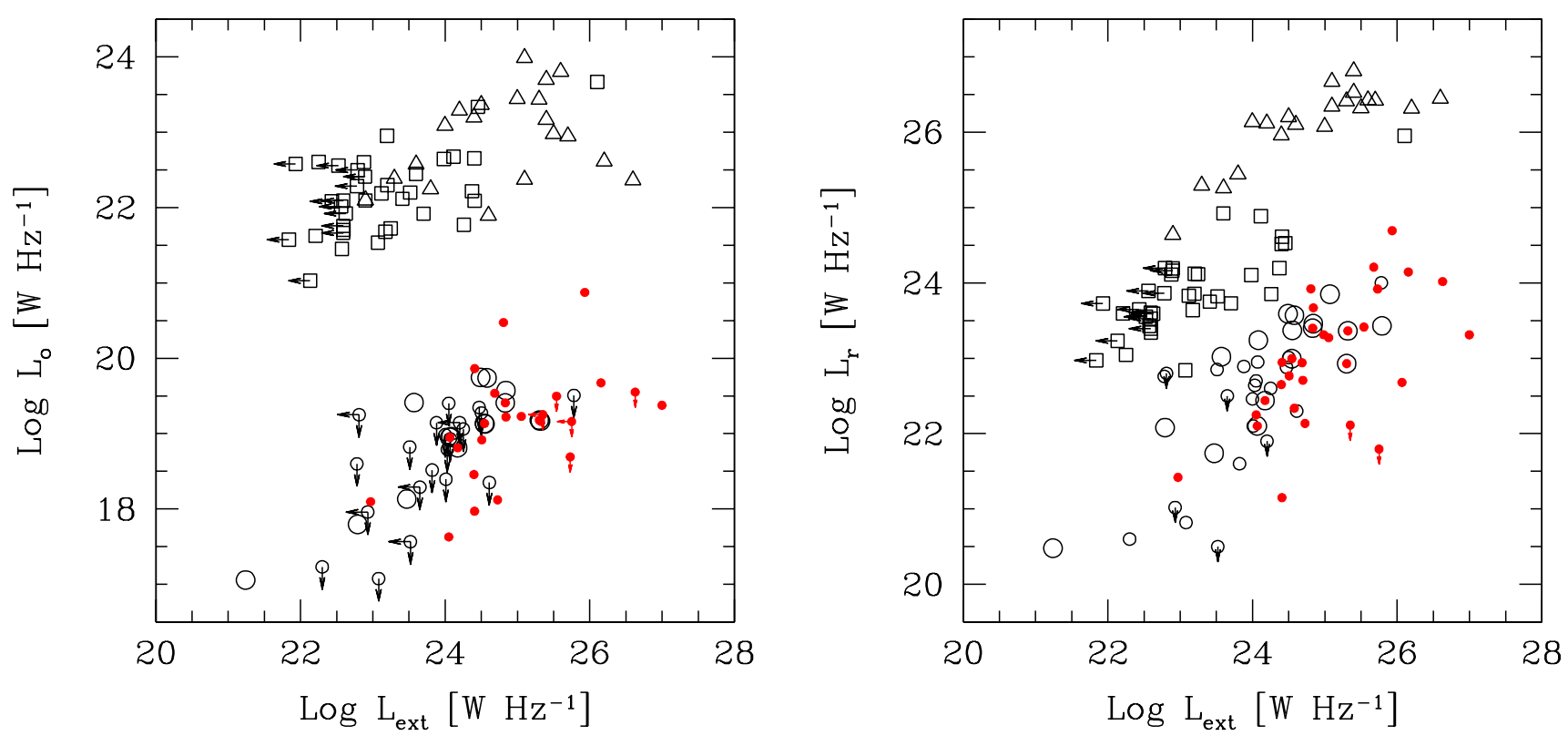

Fig. 5. Left panel: nuclear optical luminosity versus extended radio power for radio galaxies and BL Lacs. B2/FR I sources are represented by empty circles, while the filled circles are the 3C/FR I sources from Chiaberge et al. (1999). Triangles and squares are the 1 Jy and the Einstein BL Lacs samples, respectively. Right panel: same as the left panel for the core and extended radio luminosities.

from an ordinary least-squares procedure, treating upper limits as detections. Note, however, that the physical parameter of interest, the Doppler factor, that we estimate from this comparison depends only very weakly on the luminosity difference between the two classes of objects and we thus believe that the uncertainties of this procedure do not affect significantly our conclusions.

The resulting luminosity differences between FR I and $\mathrm{BL}$ Lac are $L_{\mathrm{BL}} / L_{\mathrm{FR} \mathrm{I}}=10^{2.5}$ and $L_{\mathrm{BL}} / L_{\mathrm{FR} \mathrm{I}}=$ $10^{3.9}$ in the radio and optical band respectively. We derive an average ratio of the Doppler factors between the two classes of $\delta_{\mathrm{BL}} / \delta_{\mathrm{FR} \mathrm{I}}=\left(L_{\mathrm{BL}} / L_{\mathrm{FR} \mathrm{I}}\right)^{1 /(p+\alpha)} \sim 18$ (for a radio spectral index $\alpha_{\mathrm{r}}=0$, characteristic of self-absorbed sources, and $p=2$, as appropriate if the emitting region is a continuous jet; for $p=3$, representing a moving sphere, $\left.\delta_{\mathrm{BL}} / \delta_{\mathrm{FR} \mathrm{I}} \sim 7\right)$. Similarly, in the optical band, $\delta_{\mathrm{BL}} / \delta_{\mathrm{FR} \mathrm{I}} \sim 20$ (for $\alpha_{\mathrm{o}}=1$ and $p=2$; for $p=3$, $\left.\delta_{\mathrm{BL}} / \delta_{\mathrm{FR} \mathrm{I}} \sim 9\right)$. These values are consistent with those in the radio band, i.e. the different radio and optical spectral indices account for the apparently different amplification in the two bands.

In other words this comparison indicates that the differences in luminosity between BL Lac and FR I can be explained with a single amplification factor over the whole range of extended luminosity in both the radio and optical bands. This clearly provides further support to the FR I/BL Lac unifying scheme and to the interpretation that in all cases we are seeing the synchrotron emission from a relativistic jet.

Let us now consider how the difference in Doppler factors between BL Lac and radio galaxies can be accounted for by a different viewing angle of the same relativistically beamed emission region. Assuming the same intrinsic Lorentz factor and as average viewing angles $1 / \Gamma$ and $60^{\circ}$ for $\mathrm{BL}$ Lac and FR I, respectively, we obtain $\delta_{\mathrm{BL}} / \delta_{\mathrm{FR} \mathrm{I}}=\Gamma^{2} / 2$ and therefore $\Gamma \sim 6$ for $p=2$ (for $p=3 \Gamma \sim 4)$. As already noted by CCCG00, this is significantly smaller than what inferred from observational and theoretical considerations on BL Lacs, which require $\Gamma \sim 15-20$ from both pair opacity and broad band energy distribution constraints (e.g. Dondi \& Ghisellini 1995; Ghisellini et al. 1998; Tavecchio et al. 1998). One possible interpretation of such discrepancy, as suggested by CCCG00, is that a velocity structure is present in the jet, with a fast spine (which dominates the emission in BL Lac) surrounded by a slower layer (dominant in FR I). CCC99 estimated the typical value of $\Gamma$ for the layer in the simplest (arbitrary) scenario where the two components have the same intrinsic luminosity and spectra and derived $\Gamma_{\text {layer }} \sim 1.5-2$. Laing et al. (1999) from the radio core dominance in the B2 sample of radio galaxies estimate $\Gamma_{\text {layer }} \sim 2$ and $\Gamma_{\text {spine }} \geq 9$, close to the estimate of CCC99 but with luminosity ratio $\frac{I_{\mathrm{s}}}{I_{1}} \sim 6$. These figures however are still poorly constrained by the available data.

\section{Summary and conclusions}

We discuss the optical properties of the nuclei of low luminosity radio galaxies as derived from the snapshot HST images of the B2 sample. A nuclear optical component is found in 18 out of the 57 observed galaxies and, in agreement with the results obtained from the brighter $3 \mathrm{C} / \mathrm{FR} \mathrm{I}$ sources, we found a correlation between fluxes (and luminosities) of the optical and radio cores. This provides further support to the interpretation of a synchrotron origin of the optical nuclear emission. In the sources in which we failed to detect an optical core, the optical limits are consistent with the interpretation that their nuclei follow 
the same radio/optical correlation, but their nuclear flux density is insufficient to be seen against the galaxy background. However, the large fraction of undetected CCC does not allow us to strengthen our conclusions on the lack of obscuring nuclear matter (as it was in the case of the more powerful 3CR FR I).

The radio/optical nuclear correlation for all FR I extends down to an optical luminosity of $10^{18} \mathrm{~W} \mathrm{~Hz}^{-1}$. This value can be adopted as an upper limit to any emission from the AGN not directly related to their relativistic jets, such as e.g. from the accretion disk. For a $10^{9} M_{\odot}$ central black hole, this corresponds to a fraction $\sim 3 \times 10^{-8}$ of the Eddington luminosity in the optical band and it provides a clear evidence that accretion in these low luminosity radio galaxies occurs at a very low rate and/or radiation efficiency.

In the framework of the FR I/BL Lacs unified scheme, the direct comparison of the optical nuclear properties of radio galaxies with their putative aligned counterparts provides a quantitative method of testing this unification model. The inclusion of the B2 sources significantly improves the coverage towards low extended luminosities with respect to the $3 \mathrm{C} / \mathrm{FR}$ I sample and thus it is now possible to compare the properties of the two populations over their whole range of radio power.

This comparison indicates that the differences in luminosity between BL Lac and FR I can be explained with a single amplification factor over the whole range of extended radio luminosity in both the radio and optical bands, again supporting the interpretation that in both cases we are seeing relativistic jet synchrotron emission. The corresponding bulk Lorentz factor results (for typical viewing angles) significantly smaller than derived from spectral energy properties. This support the interpretation that a velocity structure is present in the jet, with a fast spine surrounded by a slower layer where the layer is responsible for the bulk of the intrinsic emission.

Acknowledgements. The authors acknowledge the Italian MURST for financial support. This research has made use of the NASA/IPAC Extragalactic Database (NED) which is operated by the Jet Propulsion Laboratory, California Institute of Technology, under contract with the National Aeronautics and Space Administration.

\section{References}

Capetti, A., \& Celotti, A. 1999, MNRAS, 304, 434
Capetti, A., de Ruiter, H. R., Fanti, R., et al. 2000, A\&A, 362, 871, Paper I

Chiaberge, M., Capetti, A., \& Celotti, A. 1999, A\&A, 349, 77 (CCC99)

Chiaberge, M., Capetti, A., \& Celotti, A. 2000a, A\&A, 355, 873

Chiaberge, M., Celotti, A., Capetti, A., \& Ghisellini, G. 2000b, A\&A, 355, 873 (CCCG00)

Chiaberge, M., Capetti, A., \& Celotti, A. 2001, A\&A, submitted

Colla, G., Fanti, C., Fanti, R., et al. 1975, A\&AS, 20, 1

De Koff, S., Baum, S. A., Sparks, W. B., et al. 1996, ApJS, 107,621

de Ruiter, H. R., Parma, P., Capetti, A., Fanti, R., \& Morganti, R. 2001, in preparation

Dondi, L., \& Ghisellini, G. 1995, MNRAS, 273, 583

Elvis, M., Plummer, D., Schachter, J., \& Fabbiano, G. 1992, ApJS, 80, 257

Fanaroff, B. L., \& Riley, J. M. 1974, MNRAS, 167, 31

Fanti, R., Gioia, I., Lari, C., \& Ulrich, M. H. 1978, A\&AS, 34, 341

Fossati, G., Maraschi, L., Celotti, A., Comastri, A., \& Ghisellini, G. 1998, MNRAS, 299, 433

Giommi, P., \& Padovani, P. 1994, MNRAS, 268, L51

Giovannini, G., Feretti, L., Gregorini, L., \& Parma, P. 1988, A\&A, 199, 73

Giovannini, G., Cotton, W. D., Feretti, L., Lara, L., \& Venturi, T. 2001, ApJ, 552, 508

Ghisellini, G., Celotti, A., Fossati, G., Maraschi, L., \& Comastri, A. 1998, MNRAS, 301, 451

Isobe, T., \& Feigelson, E. 1985, ApJ, 293, 192

Isobe, T., \& Feigelson, E. 1986, ApJ, 306, 490

Kollgaard, R. I., Palma, C., Laurent-Muehleisen, S. A., \& Feigelson, E. D. 1996, ApJ, 465, 115

Kühr, H., Witzel, A., Pauliny-Toth, I. I. K., \& Nauber, U., 1981, A\&AS, 45, 367

Laing, R. A. 1993, Space Telescope Sci. Inst. Symp. 6, Astrophysical jets, ed. D. Bulgarella, M. Livio, \& C. P. O'Dea (Cambridge University Press), 95

Laing, R. A., Parma, P., de Ruiter, H. R., \& Fanti, R. 1999, MNRAS, 306, 513

Martel, A. R., Baum, S. A., Sparks, W. B., et al. 1999, ApJS, 122,81

Perlman, E. S., Stocke, J. T., Schachter, J. F., et al. 1996, ApJS, 104, 251

Stickel, M., Fried, J. W., Kühr, H., Padovani, P., \& Urry, C. M. 1991, ApJ, 374, 431

Tavecchio, F., Maraschi, L., \& Ghisellini, G. 1998, ApJ, 509, 608

Xu, C., Baum, S. A., O’Dea, C. P., Wrobel, J. M., \& Condon, J. J. 2000, AJ, 120, 2950 